\title{
Modified Vitamin D Binding Protein Macrophage Activator EF-022
}

National Cancer Institute

\section{Source}

National Cancer Institute. Modified Vitamin D Binding Protein Macrophage Activator EF022. NCl Thesaurus. Code C131824.

A modified version of vitamin D binding protein (VDBP; Gc protein) macrophage activator, with potential antineoplastic and anti-ang iog enic activities. Upon administration, modified VDBP-macrophage activator EF-022, acting in a similar manner as VDBP-macrophage activating factor (GcMAF), is able to activate tumoricidal macrophages, thereby enhancing the killing and eradication of cancer cells. In addition, EF-022 may inhibit tumor cell proliferation, migration and angiogenesis. VDBP is a glycoprotein and precursor for macrophage activating factor (MAF), which promotes macrophage activation; however VDBP can be deglycosylated by serum alpha- $\mathrm{N}$ acetylgalactosaminidase, which is secreted from cancerous cells, and cannot be converted to MAF. Thus, the macrophage activation cascade is often impaired in tumor cells and plays a key role in tumor immunosuppression. Modification of VDBP stabilizes MAF. 